\title{
Access, utilization, and barriers to using malaria protection tools in migrants to Iran
}

\section{Hassan Okati-Aliabad}

Health Promotion Research Center, Zahedan University of Medical Sciences, Zahedan

\section{Alireza Ansari-Moghaddam}

Health Promotion Research Center, Zahedan University of Medical Sciences, Zahedan

Mahdi Mohammadi

Health Promotion Research Center, Zahedan University of Medical Sciences, Zahedan

Jalil Nejati ( $\square$ jalilnejati@yahoo.com )

Health Promotion Research Center, Zahedan University of Medical Sciences, Zahedan

\section{Mansour Ranjbar}

Health Promotion Research Center, Zahedan University of Medical Sciences, Zahedan

\section{Ahmad Raeisi}

National Program for Malaria Control, Center of Disease Control \& Prevention, Ministry of Health and Medical Education

\section{Godarz Kolifarhood}

National Program for Malaria Control, Center of Disease Control \& Prevention, Ministry of Health and Medical Education

\section{Fariba Shahraki-Sanavi}

Health Promotion Research Center, Zahedan University of Medical Sciences, Zahedan

\section{Alireza Khorram}

Health Promotion Research Center, Zahedan University of Medical Sciences, Zahedan

\section{Research Article}

Keywords: Migrants, Malaria, Protection, Iran

Posted Date: May 19th, 2022

DOI: https://doi.org/10.21203/rs.3.rs-1378054/v2

License: (c) (i) This work is licensed under a Creative Commons Attribution 4.0 International License. Read Full License 


\section{Abstract}

Background: Imported malaria cases could be considered one of the threats to malaria elimination. Therefore, increasing migrants' access to malaria preventive measures can play an essential role in maintaining appropriate conditions and preventing malaria outbreaks. This study aimed to provide detailed information about access, utilization, and barriers to using malaria protection tools in migrants to Iran.

Methods: This study was conducted in a vast region consisting of 4 provinces and 38 cities located in the south and southeast of the country. Study participants were migrants who moved to the study area in the past three months. A sample of 4163 migrants participated in the study. They were selected through a multi-stage sampling method to obtain a representative community sample. Data were collected through interviewer-administered questionnaires about participants' socio-demographic specification, commuting characteristics, travel aim, access, ways of preparing, and reasons to use or not to use malaria protection tools. Quantitative and qualitative variables were described and analyzed finally.

Results: The mean age of individuals was $28.6 \pm 10.8$, with a range of 3-88 years old. Migrants' country of origin was Afghanistan (56.6\%), Pakistan (38.4\%), and Iran (5\%). Most migrants (69.2\%) did not have malaria protection tools while staying in Iran. Among those who prepared the protection tools, $74 \%$ used long-lasting insecticidal nets (LLINs), $13.4 \%$ used mosquito repellent sticks and coil, and $12.7 \%$ did not use any tools. Respectively, lack of knowledge about where they can get LLINs, followed by being expensive, unavailability in the market, not cooperation of health officer, and no need to use were expressed as the causes for having no access. The main reasons for non-using the tools were lack of knowledge about their application, followed by a defect in protection tools, ineffectiveness, and being harmful, respectively. Migrants who supported with an employer accessed more to LLINs.

Conclusion: This study reveals significant shortcomings in knowledge, access, and utilization of malaria protection tools among migrants in Iran. Inequitable access to public health services is predictable during migration; however, access to sustainable protection tools is recommended.

\section{Background}

Despite malaria case incidence reduction in recent years, it is still considered a major global health challenge. Some countries have started the malaria elimination program to interrupt malaria transmission and finally, no indigenous cases. Focusing on malaria protection tools is one of the most critical points in this program. In the meantime, the imported case can cause the goal not to be achieved [1].

Iran, involved with the malaria elimination program, has reduced its indigenous cases to zero in 2018 and 2019. In contrast, its eastern neighbors, Afghanistan and Pakistan, are affected by malaria where the elimination program is not implemented [2]. Afghanistan's case incidence was higher in 2020 than 2015. Although Pakistan reported a decline in the mentioned years, it was less than $40 \%$ and insignificant. The 
total results of positive microscopy and rapid diagnostic tests (RDT) for Pakistan in 2015 and 2019 were 307326 and 413533, respectively. It was 119859 and 173860 for Afghanistan. However, there was no consistent decreasing trend in the number of cases in neighboring countries in the last decade [1].

Some studies showed that imported malaria cases could be considered one of the threats that can turn the foci from clear up to residual active $[3,4]$. In recent years, almost all malaria cases in Iran have been reported as imported ones, mainly from southern and southeastern areas [5]. Sistan and Baluchestan Province (also called Sistan-Baluchestan or Sistan-va-Baluchestan) in southeastern Iran has a long shared border with Afghanistan and Pakistan. It includes the highest number of malaria cases in the country. This province is a destination for migrants from those neighboring countries annually. In addition, the southern provinces, Kerman, Hormozgan, and Bushehr, with the lower malaria incidence than the southeastern areas, are considered the next destination [6]. Due to the favorable climatic conditions for breeding malaria vectors, there is a concern for introduced and indigenous cases followed by the imported cases in this part of the country [7].

Therefore, increasing migrants' access to malaria protection tools can play an essential role in maintaining appropriate conditions and preventing malaria outbreaks [5].

There is little documentation on migrants' health challenges in Iran, and less attention has been paid to this crucial issue. This study was aimed to provide detailed information about the access, utilization, and barriers of using malaria protection tools in migrants to the country.

\section{Methods}

\section{Study area}

This cross-sectional study was conducted from April to September 2019. This investigation was done in a vast region of 4 provinces and 38 cities, located in the south and southeast of Iran (Fig. 1). The provinces were Sistan and Baluchestan (counties: Chabahar, Konarak, Saravan, Mehrestan, Sib va Sooran, Khash, Sarbaz, Iranshahr, Qasr-e-Qand, Dalgan, Fanouj, and Nikshahr), Kerman (counties: Jiroft, Anbarabad, Manoojan, Ghaleh-Ganj, Faryab, Kahnooj, and Rudbar-e-Jonub), Hormozgan (counties: Rudan, Minab, Bashagard, Bandar Lengeh, Jask, Bandar Khamir, Sirik, Hajiabad, Bastak, Bandar Abbas and Qeshm), and Bushehr (counties: Genaveh, Deylam, Tangestan, Jam, Dashtestan, Dashti, Kangan and Bushehr).

Sistan and Baluchestan Province (Lat: $25.09^{\circ} \mathrm{N}$ to $31.44^{\circ} \mathrm{N}$; Lon: $58.78^{\circ} \mathrm{E}$ to $63.26^{\circ} \mathrm{E}$ ) includes various climates. Because of proximity to the Lut desert, the northern and central regions have an arid climate with about $100 \mathrm{~mm}$ average annual rainfall. Occasionally the temperature exceeds over $50^{\circ} \mathrm{C}$ in the summer. However, Snow sometimes covers the highlands of this region. Conversely, the southern parts usually have relative humidity between $50 \%$ and $95 \%$ and heavy precipitation during the year due to monsoon currents. Overall, the average temperature is about $22^{\circ} \mathrm{C}$ to $37^{\circ} \mathrm{C}$ in this extensive province. [810]. 
The average annual precipitation for the province of Kerman (Lat: $26.03^{\circ} \mathrm{N}$ to $32.04^{\circ} \mathrm{N}$; Lon: $54.49^{\circ} \mathrm{E}$ to $59.48^{\circ} \mathrm{E}$ ) is nearly $178 \mathrm{~mm}$. However, it is about $280 \mathrm{~mm}$ per year in the southwestern regions. The average temperature is about $-16.5^{\circ} \mathrm{C}$ to $42.4^{\circ} \mathrm{C}$ in this largest Iranian province [11].

Hormozgan Province (Lat: $25.23^{\circ} \mathrm{N}$ to $28.97^{\circ} \mathrm{N}$; Lon: $52.41^{\circ} \mathrm{E}$ to $59.15^{\circ} \mathrm{E}$ ) has arid and semiarid climates. The average annual rainfall is much less than $250 \mathrm{~mm}$, which causes intense flooding. Temperatures sometimes reach $49^{\circ} \mathrm{C}$ in the summer. Nevertheless, like the southern parts of Sistan and Baluchestan Province, the weather along the coast is mild in the winter [12].

Bushehr Province is located in the southwest of Iran (Lat: $27.39^{\circ} \mathrm{N}$ to $30.25^{\circ} \mathrm{N}$; Lon: $50.13^{\circ} \mathrm{E}$ to $52.96^{\circ} \mathrm{E}$ ). With an average annual rainfall of about $230 \mathrm{~mm}$ and $24^{\circ} \mathrm{C}$ average temperatures, this region has an arid and subtropical climate [13].

Numerous studies have shown that these areas are suitable for mosquitoes breeding due to the favorable climate and monsoon currents, especially in the southeastern parts [10,14]. In addition, five of the seven malaria vectors in Iran have been established and can be collected in these areas[5].

The study areas were selected based on published papers on the high risk of malaria and increased migration. In addition, undocumented evidence through interviews with local malaria experts was used.

\section{Study participants and sampling}

Study participants were migrants who moved to the study area in the past three months for temporary farming, service work, fishing, aquaculture, etc. A sample of 4163 migrants participated in the study. They were selected through a multi-stage sampling method to obtain a representative community sample. In the 1 st stage, we used purposive sampling to include counties in each province with significant numbers of migrants. At the 2 nd stage, the main areas for the residence of the migrants in each county and the number of migrants in each area were identified. At the last step, the areas and the number of people in each region were selected proportional to the size and a simple random sampling method.

Inclusion criteria were migration to Iran in the last three months and informed consent to participate in the study. Individuals with severe physical or mental illness who could not participate were excluded from the study.

\section{Data collection}

Data were collected through interviewer-administered questionnaires. The interviewers were local health workers who speak and understand Persian and Urdu languages. Data collection tools were developed after reviewing the relevant literature and meetings with malaria experts at the Center for Communicable Disease Control in the Ministry of Health and Medical Education of Iran and malaria experts at the Zahedan University of Medical Sciences. Data were collected on participants' socio-demographic 
specification, commuting characteristics, travel aim, access, ways of preparing, and reasons to use or not to use malaria protection tools.

The research team held a briefing session and a training workshop for interviewers in all provinces.

Training content included stating the purpose of the study, explaining the different parts of the questionnaire, the role of the interviewer and the supervisor, how to sample the study sites and participants, how to communicate with participants, seeking informed consent, interview techniques, the confidentiality of information, checking and sending completed questionnaires.

\section{Data analysis}

Data were statistically analysed using Statistical Package for Social Science version 24 software (IBM SPSS Statistics for Windows). Quantitative and qualitative variables were described as mean \pm standard deviation, number (percent), and odds ratio (OR), respectively. Furthermore, the chi-square test was used to determine the relationship between qualitative variables. Levels of significance were set at $P<.05$.

\section{Results}

In total, 4163 migrants to Iran were investigated. The mean age of individuals was $28.6 \pm 10.8$, with a range of 3-88 years old. The majority of individuals were male (87.6\%) and married (68.8\%). Migrants' country of origin was Afghanistan (56.6\%), Pakistan (38.4\%), and Iran (5\%). The permanent living place of migrants was Afghanistan (61.3\%), Pakistan (37.9\%), and other countries (0.8\%) (Table 1).

Most migrants (61.7\%) crossed the border once, and $11.8 \%$ commuted more than three times a year. About half of migrants planned to stay in Iran for less than four months and $27 \%$ more than six months. Afghan migrants crossed the border fewer times with more extended stays in Iran (Table 1). 
Table 1

\begin{tabular}{|c|c|c|}
\hline Criteria & Item & $\mathrm{n}(\%)$ \\
\hline \multirow[t]{6}{*}{ Age (year) } & $\leq 15$ & $182(4.4)$ \\
\hline & $16-20$ & $867(20.9)$ \\
\hline & $21-25$ & $918(22.1)$ \\
\hline & $26-30$ & $835(20.1)$ \\
\hline & $31-40$ & $815(19.6)$ \\
\hline & More than 40 & $535(12.9)$ \\
\hline \multirow[t]{3}{*}{ Gender } & Male & $3615(87.6)$ \\
\hline & Female (non-pregnant) & $479(11.6)$ \\
\hline & Female (pregnant) & $33(0.8)$ \\
\hline \multirow[t]{3}{*}{ Marriage } & Single & $1282(31.0)$ \\
\hline & Married & $2847(68.8)$ \\
\hline & Widow & $11(0.2)$ \\
\hline \multirow[t]{3}{*}{ Nationality } & Iran & $204(5)$ \\
\hline & Pakistan & $1583(38.4)$ \\
\hline & Afghanistan & $2335(56.6)$ \\
\hline \multirow[t]{3}{*}{ Permanent living place } & Pakistan & $1491(37.9)$ \\
\hline & Afghanistan & $2416(61.3)$ \\
\hline & Others & $31(0.8)$ \\
\hline \multirow[t]{3}{*}{ Number of times crossing the border in a year } & 1 & $2395(61.7)$ \\
\hline & $2-3$ & $1030(26.5)$ \\
\hline & $>3$ & $458(11.8)$ \\
\hline \multirow[t]{4}{*}{ Duration staying in Iran on the last trip (month) } & 1 & $1056(29.2)$ \\
\hline & $2-3$ & $862(23.8)$ \\
\hline & $4-6$ & $717(19.8)$ \\
\hline & $>6$ & 981 (27.2) \\
\hline
\end{tabular}


Regardless of the type of employment, most working migrants were male, married, Afghani, aged 16-30 years, lived permanently in Afghanistan, and stayed temporarily in Iran. Most non-working migrants were Pakistani and 16-30 years old. The frequency distribution of demographic characteristics was significantly different among non-working, with, and without employer migrants (Table 2).

In Iran, $59.7 \%$ of migrants lived in a temporary place, and $50.8 \%$ had to change their homes more than once annually. Only $18 \%$ lived in a fully-finished building, while $40.1 \%$ lived in a shared room with their colleagues and $14.2 \%$ in a friend's house. About $28 \%$ stayed in unequipped and inappropriate places.

Table 2

Frequency distribution of travel aim in terms of demographic characteristics

\begin{tabular}{|c|c|c|c|c|c|}
\hline \multirow[t]{2}{*}{ Criteria } & \multirow[t]{2}{*}{ Item } & \multicolumn{2}{|l|}{ Working trip } & \multirow{2}{*}{$\begin{array}{l}\text { Non-working } \\
\text { trip }\end{array}$} & \multirow{2}{*}{$\begin{array}{l}P_{-} \\
\text {value }\end{array}$} \\
\hline & & $\begin{array}{l}\text { With } \\
\text { employer }\end{array}$ & $\begin{array}{l}\text { Without } \\
\text { employer }\end{array}$ & & \\
\hline \multirow[t]{2}{*}{ Gender } & Male & 1878 (97.5) & 1240 (88.9) & $451(60.1)$ & \multirow[t]{2}{*}{$<0.001$} \\
\hline & Female & $48(2.5)$ & 155 (11.1) & $299(39.9)$ & \\
\hline \multirow[t]{2}{*}{ Marriage } & Single & $681(35.1)$ & $416(29.8)$ & $168(22.4)$ & \multirow[t]{2}{*}{$<0.001$} \\
\hline & Married/widow & $1256(64.9)$ & 982 (70.2) & $573(77.6)$ & \\
\hline \multirow[t]{3}{*}{ Nationality } & Iranian & $43(2.2)$ & $60(4.3)$ & $101(13.4)$ & \multirow[t]{3}{*}{$<0.001$} \\
\hline & Pakistani & 764 (39.7) & $430(31.0)$ & $380(50.4)$ & \\
\hline & Afghan & $1118(58.1)$ & 897 (64.7) & $273(36.2)$ & \\
\hline \multirow{2}{*}{$\begin{array}{l}\text { Permanent } \\
\text { place }\end{array}$} & Pakistan & 754 (39.9) & 362 (27.5) & 367 (56.9) & \multirow[t]{2}{*}{$<0.001$} \\
\hline & Afghanestan & $1137(60.1)$ & 954 (72.5) & $278(43.1)$ & \\
\hline \multirow[t]{2}{*}{ Type of stay } & Permanent & 715 (36.9) & 632 (45.3) & 307 (40.4) & \multirow[t]{2}{*}{$<0.001$} \\
\hline & Temporary & $1222(63.1)$ & 764 (54.7) & $452(59.6)$ & \\
\hline \multirow[t]{4}{*}{ Age (year) } & $<=15$ & $49(2.5)$ & $62(4.4)$ & $67(8.9)$ & \multirow[t]{4}{*}{$<0.001$} \\
\hline & $16-30$ & $1386(71.3)$ & 864 (61.9) & $334(44.2)$ & \\
\hline & $31-50$ & $471(24.3)$ & $396(28.3)$ & $275(36.3)$ & \\
\hline & $>50$ & $36(1.9)$ & $76(5.4)$ & $80(10.6)$ & \\
\hline
\end{tabular}

Most migrants did not have access to malaria protection tools during staying in Iran (69.2\%). Some migrants had access to long-lasting insecticidal nets (LLINs) (26\%), mosquito repellent [16] stick (3.4\%), and coil (1.4\%). Compared to Pakistani migrants, Afghan migrants had more access to MR stick (OR = 1.94) and coil $(O R=5.46)$ and less access to LLINs $(O R=0.45)$ in their accommodation (Table 3$)$. 
Most migrants prepared the protection tools either through the health care system (64.4\%) or by buying personally $(20.6 \%)$. The chance of purchasing protection tools $(O R=4.39)$ and receiving from local people $(O R=1.70)$ was more in Afghan than Pakistani migrants, while the opportunity of preparing through the health care system was less in Afghan migrants $(O R=0.41)($ Table 3$)$. 
Table 3

Frequency distribution of access and use of malaria protection tools in migrants

\begin{tabular}{|c|c|c|c|c|c|}
\hline Criteria & Item & $N(\%)$ & Pakistan & Afghanistan & $\begin{array}{l}\text { OR (Af } \\
\text { vs.Pa) }\end{array}$ \\
\hline \multirow[t]{4}{*}{$\begin{array}{l}\text { Malaria protection tools access } \\
\text { in the current accommodation }\end{array}$} & LLINs & $\begin{array}{l}1074 \\
(26.0)\end{array}$ & $\begin{array}{l}525 \\
(35.2)\end{array}$ & 475 (19.8) & $\begin{array}{l}0.45(0.39 \\
0.52)\end{array}$ \\
\hline & MR stick & $\begin{array}{l}142 \\
(3.4)\end{array}$ & $33(2.2)$ & $101(4.2)$ & $\begin{array}{l}1.94 \\
(1.30,2.88)\end{array}$ \\
\hline & MR coil & $\begin{array}{l}59 \\
(1.4)\end{array}$ & $6(0.4)$ & $52(2.2)$ & $\begin{array}{l}5.46 \\
(2.34,12.75)\end{array}$ \\
\hline & None & $\begin{array}{l}2877 \\
(69.2)\end{array}$ & $\begin{array}{l}930 \\
(62.2)\end{array}$ & 1772 (73.8) & $\begin{array}{l}0.59 \\
(0.51,0.68)\end{array}$ \\
\hline \multirow{9}{*}{$\begin{array}{l}\text { Given access, ways of } \\
\text { preparing malaria protection } \\
\text { tools }\end{array}$} & $\begin{array}{l}\text { Bought by } \\
\text { the person }\end{array}$ & $\begin{array}{l}256 \\
(20.6)\end{array}$ & $51(9.5)$ & $190(31.6)$ & $\begin{array}{l}4.39 \\
(3.14,6.14)\end{array}$ \\
\hline & Employer & $\begin{array}{l}59 \\
(4.7)\end{array}$ & $29(5.4)$ & $29(4.8)$ & $\begin{array}{l}0.89 \\
(0.53,1.51)\end{array}$ \\
\hline & Health center & $\begin{array}{l}800 \\
(64.4)\end{array}$ & $\begin{array}{l}410 \\
(76.5)\end{array}$ & $332(55.1)$ & $\begin{array}{l}0.41 \\
(0.32,0.52)\end{array}$ \\
\hline & Local people & $\begin{array}{l}23 \\
(1.8)\end{array}$ & $8(1.5)$ & $15(2.5)$ & $\begin{array}{l}1.70 \\
(0.72,4.04)\end{array}$ \\
\hline & $\begin{array}{l}\text { Traditional } \\
\text { healers }\end{array}$ & $\begin{array}{l}2 \\
(0.2)\end{array}$ & $0(0.0)$ & $2(0.3)$ & - \\
\hline & Friend's gift & $\begin{array}{l}18 \\
(1.4)\end{array}$ & $13(2.4)$ & $5(0.8)$ & $\begin{array}{l}0.34 \\
(0.12,0.96)\end{array}$ \\
\hline & $\begin{array}{l}\text { Neighbor's } \\
\text { gift }\end{array}$ & $\begin{array}{l}5 \\
(0.4)\end{array}$ & $3(0.6)$ & $2(0.3)$ & $\begin{array}{l}0.60 \\
(0.10,3.59)\end{array}$ \\
\hline & Others & $\begin{array}{l}28 \\
(2.3)\end{array}$ & - & - & - \\
\hline & $\begin{array}{l}\text { A } \\
\text { combination } \\
\text { of items }\end{array}$ & $\begin{array}{l}53 \\
(4.2)\end{array}$ & $22(4.1)$ & $28(4.6)$ & - \\
\hline \multirow{4}{*}{$\begin{array}{l}\text { Given access, malaria } \\
\text { protection tools used in the } \\
\text { accommodation }\end{array}$} & LLINs & $\begin{array}{l}929 \\
(73.9)\end{array}$ & $\begin{array}{l}480 \\
(87.1)\end{array}$ & $392(62.7)$ & $\begin{array}{l}0.27 \\
(0.20,0.36)\end{array}$ \\
\hline & MR stick & $\begin{array}{l}116 \\
(9.2)\end{array}$ & $17(3.1)$ & $91(14.6)$ & $\begin{array}{l}5.43 \\
(3.19,9.25)\end{array}$ \\
\hline & MR coil & $\begin{array}{l}53 \\
(4.2)\end{array}$ & $4(0.7)$ & $49(7.9)$ & $\begin{array}{l}11.80 \\
(4.23,32.92)\end{array}$ \\
\hline & None & $\begin{array}{l}160 \\
(12.7)\end{array}$ & $50(9.1)$ & $93(14.8)$ & $\begin{array}{l}1.77 \\
(1.23,2.55)\end{array}$ \\
\hline
\end{tabular}


The using status among migrants who could prepare the protection tools was $73.9 \%$ LLINs, $13.4 \%$ MR stick and coils, and $12.7 \%$ did not use any tools. Pakistani migrants more used LLINs, while MR stick and coils were more likely to be used by Afghan migrants (Table 3).

The reasons for non-using the tools were as follows; lack of knowledge about applying the tools (21.2\%), defects in protection tools (16.2\%), ineffectiveness (9.1\%), and being harmful (2.8\%). Migrants' motives for lack of using protection tools were different. Afghan migrants believed less in ineffectiveness $(\mathrm{OR}=$ $0.44)$ but more in harmfulness $(O R=2.78)$ of the tools than Pakistani migrants (Table 4$)$.

The reasons for having no access were as follows; they did not know where to get the tools $(57.2 \%)$, unavailability in the market (7.8\%), being expensive (14.2\%), lack of health officer cooperation (5.9\%), and no need to use $(0.6 \%)$. Lack of knowledge about the way obtaining the protection tools was more prevalent among Afghan than Pakistani migrants $(O R=1.41)$. Compared to Pakistani migrants, Afghans had fewer complaints about the unavailability of tools on the market $(\mathrm{OR}=0.74)$, being expensive $(\mathrm{OR}=$ $0.71)$, and preparing through the health care system $(\mathrm{OR}=0.5)($ Table 4$)$. 
Table 4

Frequency distribution of non-access and no use of malaria protection tools in migrants

\begin{tabular}{|c|c|c|c|c|c|}
\hline Criteria & Item & $\mathbf{N}(\%)$ & Pakistan & Afghanistan & OR (Af vs.Pa) \\
\hline \multirow{4}{*}{$\begin{array}{l}\text { Given not using, reasons } \\
\text { not to use malaria } \\
\text { protection tools in the } \\
\text { current accommodation }\end{array}$} & $\begin{array}{l}\text { Ineffective in } \\
\text { preventing } \\
\text { infection }\end{array}$ & $\begin{array}{l}112 \\
(9.1)\end{array}$ & $\begin{array}{l}73 \\
(13.1)\end{array}$ & 37 (6.2) & $\begin{array}{l}0.44 \\
(0.29,0.66)\end{array}$ \\
\hline & $\begin{array}{l}\text { Do not know } \\
\text { how to apply the } \\
\text { tools }\end{array}$ & $\begin{array}{l}261 \\
(21.2)\end{array}$ & $\begin{array}{l}135 \\
(24.2)\end{array}$ & $120(20.1)$ & $\begin{array}{l}0.79 \\
(0.60,1.04)\end{array}$ \\
\hline & Harmful & $\begin{array}{l}35 \\
(2.8)\end{array}$ & $9(1.6)$ & $26(4.4)$ & $2.78(1.29,5.98)$ \\
\hline & $\begin{array}{l}\text { Defect in } \\
\text { protection tools }\end{array}$ & $\begin{array}{l}200 \\
(16.2)\end{array}$ & $\begin{array}{l}119 \\
(21.3)\end{array}$ & $72(12.1)$ & $\begin{array}{l}0.51 \\
(0.37,0.70)\end{array}$ \\
\hline \multirow[t]{4}{*}{$\begin{array}{l}\text { Reasons for not } \\
\text { accessing malaria } \\
\text { protection tools during } \\
\text { staying in the current } \\
\text { accommodation }\end{array}$} & $\begin{array}{l}\text { I do not know } \\
\text { where to get } \\
\text { malaria } \\
\text { protection tools }\end{array}$ & $\begin{array}{l}2190 \\
(57.2)\end{array}$ & $\begin{array}{l}763 \\
(54.0)\end{array}$ & $1362(62.3)$ & $\begin{array}{l}1.41(1.23, \\
1.61)\end{array}$ \\
\hline & $\begin{array}{l}\text { Not available in } \\
\text { the market }\end{array}$ & $\begin{array}{l}297 \\
(7.8)\end{array}$ & $\begin{array}{l}135 \\
(9.6)\end{array}$ & $158(7.2)$ & $\begin{array}{l}0.74 \\
(0.58,0.94)\end{array}$ \\
\hline & $\begin{array}{l}\text { Expensive, I } \\
\text { cannot afford to } \\
\text { buy }\end{array}$ & $\begin{array}{l}545 \\
(14.2)\end{array}$ & $\begin{array}{l}242 \\
(17.1)\end{array}$ & $280(12.8)$ & $\begin{array}{l}0.71 \\
(0.59,0.86)\end{array}$ \\
\hline & $\begin{array}{l}\text { I asked form } \\
\text { health system } \\
\text { but the health } \\
\text { officer did not } \\
\text { give it to me }\end{array}$ & $\begin{array}{l}225 \\
(5.9)\end{array}$ & $\begin{array}{l}106 \\
(7.5)\end{array}$ & $85(3.9)$ & $\begin{array}{l}0.50 \\
(0.37,0.67)\end{array}$ \\
\hline
\end{tabular}

Most migrants traveled to work in Iran (82.9\%), of which $58.1 \%$ worked under the supervision of an employer. Lack of access to malaria protection tools was more in migrants without an employer $(75 \%)$ than in other groups $(P<0.001)$. Generally, access to MR stick or coil was low. Migrants who supported with an employer accessed more to LLINs (34.1\%) compared to those with no employer (18.4\%) and nonworking migrants $(19.9 \%)(\mathrm{P}<0.001)$. About one-third of migrants without an employer and non-working ones had prepared the tools themselves compared to $11.4 \%$ of employed migrants $(P<0.001)$. About the reasons for not using the protection tools, $25.2 \%$ of employed workers and $12.5 \%$ of workers without employers reported that they did not know how to apply these tools $(P<0.001)$. More than half of migrants did not know where to get malaria protection tools $(P=0.545)$ (Tables 5 and 6$)$. 
Table 5

Frequency distribution of aim of travel in terms of access and use of malaria protection tools

\begin{tabular}{|c|c|c|c|c|c|}
\hline \multirow[t]{2}{*}{ Criteria } & \multirow[t]{2}{*}{ Item } & \multicolumn{2}{|c|}{ working trip } & \multirow{2}{*}{$\begin{array}{l}\text { Non- } \\
\text { working } \\
\text { trip }\end{array}$} & \multirow{2}{*}{$\begin{array}{l}P_{-} \\
\text {value }\end{array}$} \\
\hline & & $\begin{array}{l}\text { With } \\
\text { employer }\end{array}$ & $\begin{array}{l}\text { Without } \\
\text { employer }\end{array}$ & & \\
\hline \multirow[t]{4}{*}{$\begin{array}{l}\text { Malaria protection tools access in the } \\
\text { current accommodation }\end{array}$} & LLINs & $\begin{array}{l}651 \\
(34.1)\end{array}$ & $\begin{array}{l}260 \\
(18.4)\end{array}$ & $\begin{array}{l}157 \\
(19.9)\end{array}$ & $\begin{array}{l}< \\
0.001\end{array}$ \\
\hline & MR stick & $33(1.7)$ & $61(4.3)$ & $46(5.8)$ & $\stackrel{<}{0.001}$ \\
\hline & MR coil & $8(0.4)$ & $33(2.3)$ & $18(2.4)$ & $\begin{array}{l}< \\
0.001\end{array}$ \\
\hline & None & $\begin{array}{l}1219 \\
(63.8)\end{array}$ & $\begin{array}{l}1056 \\
(75.0)\end{array}$ & $\begin{array}{l}568 \\
(71.9)\end{array}$ & $\begin{array}{l}< \\
0.001\end{array}$ \\
\hline \multirow[t]{9}{*}{$\begin{array}{l}\text { Given access, ways of preparing } \\
\text { malaria protection tools }\end{array}$} & $\begin{array}{l}\text { Bought by } \\
\text { the person }\end{array}$ & $81(11.4)$ & $\begin{array}{l}106 \\
(31.5)\end{array}$ & $\begin{array}{l}68 \\
(35.8)\end{array}$ & $\begin{array}{l}< \\
0.001\end{array}$ \\
\hline & Employer & $49(6.9)$ & - & - & - \\
\hline & Health center & $\begin{array}{l}536 \\
(75.4)\end{array}$ & $\begin{array}{l}175 \\
(52.1)\end{array}$ & $\begin{array}{l}87 \\
(45.8)\end{array}$ & $\dot{c}_{0.001}$ \\
\hline & Local people & $3(0.4)$ & $15(4.5)$ & $5(2.6)$ & $\begin{array}{l}< \\
0.001\end{array}$ \\
\hline & $\begin{array}{l}\text { Traditional } \\
\text { healers }\end{array}$ & $0(0.0)$ & $1(0.3)$ & $0(0.0)$ & 0.261 \\
\hline & Friend's gift & $8(1.1)$ & $2(0.6)$ & $8(4.2)$ & 0.002 \\
\hline & $\begin{array}{l}\text { Neighbor's } \\
\text { gift }\end{array}$ & $2(0.3)$ & $2(0.6)$ & $1(0.5)$ & 0.726 \\
\hline & Others & $15(2.1)$ & $8(2.4)$ & $4(2.1)$ & \\
\hline & $\begin{array}{l}\text { A } \\
\text { combination } \\
\text { of items }\end{array}$ & $17(2.4)$ & $27(8.0)$ & $17(9.0)$ & $\begin{array}{l}< \\
0.001\end{array}$ \\
\hline \multirow[t]{4}{*}{$\begin{array}{l}\text { Given access, malaria protection tools } \\
\text { used in the accommodation }\end{array}$} & LLINs & $\begin{array}{l}566 \\
(83.7)\end{array}$ & $\begin{array}{l}224 \\
(65.7)\end{array}$ & $\begin{array}{l}135 \\
(70.7)\end{array}$ & $\begin{array}{l}< \\
0.001\end{array}$ \\
\hline & MR stick & $18(2.6)$ & $49(14.4)$ & $\begin{array}{l}47 \\
(24.7)\end{array}$ & $\dot{0} 001$ \\
\hline & MR coil & $7(1.0)$ & $33(9.7)$ & $13(6.8)$ & $\dot{0} 001$ \\
\hline & None & $86(12.7)$ & 47 (13.7) & $\begin{array}{l}25 \\
(13.2)\end{array}$ & 0.714 \\
\hline
\end{tabular}


Table 6

Frequency distribution of aim of travel in terms of non- access and no use of malaria protection tools

\begin{tabular}{|c|c|c|c|c|c|}
\hline \multirow[t]{2}{*}{ Criteria } & \multirow[t]{2}{*}{ Item } & \multicolumn{2}{|c|}{ working trip } & \multirow{2}{*}{$\begin{array}{l}\text { Non- } \\
\text { working } \\
\text { trip }\end{array}$} & \multirow{2}{*}{$\begin{array}{l}P_{-} \\
\text {value }\end{array}$} \\
\hline & & $\begin{array}{l}\text { With } \\
\text { employer }\end{array}$ & $\begin{array}{l}\text { Without } \\
\text { employer }\end{array}$ & & \\
\hline \multirow{4}{*}{$\begin{array}{l}\text { Given not using, reasons not to } \\
\text { use malaria protection tools in } \\
\text { the current accommodation }\end{array}$} & $\begin{array}{l}\text { Ineffective in } \\
\text { preventing infection }\end{array}$ & $91(12.7)$ & $16(4.9)$ & $5(2.7)$ & $\begin{array}{l}< \\
0.001\end{array}$ \\
\hline & $\begin{array}{l}\text { Do not know how to } \\
\text { apply the tools }\end{array}$ & $\begin{array}{l}180 \\
(25.2)\end{array}$ & $41(12.5)$ & $\begin{array}{l}38 \\
(20.9)\end{array}$ & $\begin{array}{l}< \\
0.001\end{array}$ \\
\hline & Harmful & $10(1.4)$ & $20(6.1)$ & $5(2.7)$ & $\begin{array}{l}< \\
0.001\end{array}$ \\
\hline & $\begin{array}{l}\text { Defect in protection } \\
\text { tools }\end{array}$ & $\begin{array}{l}114 \\
(16.0)\end{array}$ & $54(16.5)$ & $\begin{array}{l}32 \\
(17.6)\end{array}$ & 0.868 \\
\hline \multirow{4}{*}{$\begin{array}{l}\text { Reasons for not accessing } \\
\text { malaria protection tools during } \\
\text { staying in the current } \\
\text { accommodation }\end{array}$} & $\begin{array}{l}\text { I do not know where } \\
\text { to get malaria } \\
\text { protection tools }\end{array}$ & $\begin{array}{l}1044 \\
(56.9)\end{array}$ & $\begin{array}{l}701 \\
(56.6)\end{array}$ & $\begin{array}{l}427 \\
(59.0)\end{array}$ & 0.545 \\
\hline & $\begin{array}{l}\text { Not available in the } \\
\text { market }\end{array}$ & $157(8.6)$ & 79 (6.4) & 59 (8.1) & 0.080 \\
\hline & $\begin{array}{l}\text { Expensive, I cannot } \\
\text { afford to buy }\end{array}$ & $\begin{array}{l}202 \\
(11.0)\end{array}$ & $\begin{array}{l}239 \\
(19.3)\end{array}$ & $\begin{array}{l}99 \\
(13.7)\end{array}$ & $\begin{array}{l}< \\
0.001\end{array}$ \\
\hline & $\begin{array}{l}\text { I asked form health } \\
\text { system but the } \\
\text { health officer did not } \\
\text { give it to me }\end{array}$ & $96(5.2)$ & 85 (6.9) & $44(6.1)$ & 0.168 \\
\hline
\end{tabular}

\section{Discussion}

Iran is one of the countries with the highest number of migrants globally [15]. It has resulted previously; most malaria cases in Iran were attributed to Sistan and Baluchestan Province, mainly along the border with Pakistan. Most of them were imported cases, the citizens of Pakistan and then Afghanistan [16]. So an investigation on the health challenges of migrants can be considered as an essential issue.

Unlike previous, most migrants were Afghans in the present study, and some of them entered Iran via Pakistan. It was documented that many Afghan migrants pass through Pakistani malarious areas. Most Pakistani migrants to Iran are from high-risk malaria areas with poor welfare and care services near the shared border [17]. Therefore, their access to malaria protection tools is vital to maintaining the community's health [18].

Social, cultural, linguistic, and religious ties between Afghanistan and Pakistan and Iran, especially in the areas close to the border, and one million registered and 1.5 million illegal Afghan migrants in Iran, lead to close relationship and mobility of people crossing the border [19]. The main motivations for migrants are 
seeking job opportunities, trade, visiting friends and relatives, attending traditional or cultural ceremonies, and going on a pilgrimage [20]. It seems these reasons can lead to their frequent cross-border traffic throughout the year. Some migrants had traveled more than three times a year in the present study. This migratory movement to malarious areas will increase the possibility of their infection and the occurrence of introduced cases in the country [21].

This study showed that most migrants have traveled to Iran for work. In many studies, it has been cited as the main reason for migration. Due to migrants working in malaria-endemic areas and their lack of knowledge about its transmission and protection usually have a higher prevalence of malaria than the resident population [22]. It seems that creating the proper infrastructure to provide health services to migrants is essential for their health and the host country's people.

Most migrants lived in temporary accommodation in the current study, and about $28 \%$ of them lived in unequipped and inappropriate places. Although this situation has been reported for migrants in some other countries, its improvement can play an essential role in preventing malaria outbreaks[23, 24]. Numerous studies have shown that access to suitable accommodation with welfare amenities such as electricity, air conditioner, etc., especially at night, can reduce mosquito densities and malaria transmission $[14,25]$.

In the meantime, access to malaria protection tools is even more critical. Unfortunately, the current study found that most migrants have no access to them. Although there is a policy of distributing free LLINs in the malaria elimination program in Iran [26], it seems that this protection tools do not cover all areas. However, migrants who had access to malaria protection tools; stated that they received it from the health system. In our study, Afghans had more access to MR stick and coil than Pakistani. At the same time, they were more likely to buy malaria protection tools. Perhaps the reason is the possibility of more accessible verbal communication and cultural match that facilitate the fulfillment of daily needs[27].

Using LLINs as one of the most critical malaria protection tools is recommended by the World Health Organization. Extensive LLINs in a malarious area can significantly reduce malaria [28]. Our study showed that LLINs were the main malaria protection tools among migrants, although it was not easily accessible. Similarly, it has been documented as the primary protection tool among migrants in some countries[29].

In the present study, insufficient knowledge about the protection tools led to non-use. This issue has also been reported in several studies [23]. A study conducted in Ethiopia resulted in education significantly associated with the knowledge and practice of malaria protection tools. This result can further highlight the role of health education in preventing malaria outbreaks caused by migrants' traffic [30]. Although health education includes how to use malaria protection tools, it seems that the ways to provide them should also be informed. The current investigation showed some migrants did not know where they could get them. Raising awareness of migrant families about appropriate malaria prevention services has been suggested as essential in primary health care. In a study in the United Kingdom, practitioners and health workers highlighted providing tailored messages on preventing malaria that could have a considerable 
impact on malaria [31]. Generally, the elimination program should address equitable access to malaria preventive measures [32].

According to the present study results, access to malaria protection tools was higher among migrants who have employers that can be considered a capacity to promote the health of migrants. For instance, distribution of educational media among them can be done by employers. An investigation on Asian migrants to Angola showed most of them had been received information on malaria and its chemoprophylaxis from their employers. Even migrants with febrile illness prefer to seek care through their employer than in the public health system [33].

In our study, access to MR stick or coil was less. It seems; they are not free tools. Although some of them, like diethyl-3-methylbenzamide (DEET) used in national research, no document was found on their free distribution by the health system [34]. A study conducted on migrant populations in Myanmar showed $\mathrm{N}, \mathrm{N}$-diethyl-benzamide as MR stick has an influential role in reducing the incidence of $P$. falciparum and $P$. vivax infections. Inequitable access to public health services is predictable during migration; however, access to appropriate protection tool is considered a universal approach [35].

Migrants with an employer had more access to LLINs as a critical tool to prevent malaria. It seems employers have been involved in informing the health system to provide free health services. In other words, due to the lack of health system information on the situation of migrants without an employer, they have been forced to buy other tools instead of free LLINs, such as MR stick. Similarly, unregistered migrants were seldom achieved by LLIN-distribution campaigns in Cambodia. Actually, they were uninformed of the village malaria workers system due to poor social integration [18]. In this status, it is recommended to use the capacity of health volunteers. The experience conducted in Iran showed that volunteers play an essential role in providing health services to the people. More than 3,700 trained health volunteers were used to perform rapid diagnostic tests (RDT) among unauthorized refugees. This capacity has been considered a significant advance towards eliminating malaria in Iran [36].

\section{Conclusions}

This study reveals significant shortcomings in knowledge, access, and utilization of malaria protection tools among migrants in Iran. We recommend qualitative research for a better understanding of this issue. The enhanced awareness campaigns and planning to increase the migrant's access to the malaria protection tools are also proposed. Strengthening migrants' and employers' awareness and capacity building to facilitate access to those tools will help eliminate malaria.

\section{Abbreviations}

LLINs: long-lasting insecticidal nets; MR: mosquito repellent.

\section{Declarations}




\section{Acknowledgements}

The authors are grateful to the malaria experts at the Center for Communicable Disease Control in the Ministry of Health and Medical Education of Iran and the Zahedan University of Medical Sciences for their technical support. We appreciate all the participants for their voluntary participation. Also, we are grateful to the health care workers in each township who participated in data collection and the malaria officials in each township who were instrumental in providing oversight during data collection. Finally, we would like to thank the Health Deputy of Zahedan University of Medical Sciences, Iranshahr University of Medical Sciences, Jiroft University of Medical Sciences, Hormozgan University of Medical Sciences and Bushehr University of Medical Sciences for their logistic support.

\section{Authors' contributions}

HOA, AAM, JN, MM, MR, AR, GK, FSS, and AK conceptualized and designed the study.

HOA, AAM, FSS, and AK directed the study implementation.

MR, AR, GK supervised the study.

AAM and MM analyzed and interpreted the data.

HOA, MM, and JN drafted the manuscript.

HOA and JN revised the manuscript for important intellectual content.

All the authors have read and approved the final manuscript.

\section{Funding}

This study was supported by the Center for Communicable Disease Control in the Ministry of Health and Medical Education of Iran.

\section{Availability of data and materials}

The datasets used and/or analyzed during the current study are available from the corresponding author on reasonable request.

\section{Ethics approval and consent to participate}

This study was approved by the Ethics Review Committee of Zahedan University of Medical Sciences (IR.ZAUMS.REC.1395.246). The study was conducted in accordance with the Declaration of Helsinki and verbal informed consent was obtained from all study participants.

\section{Consent for publication}

Not applicable. 


\section{Competing interests}

The authors declare that they have no competing interests.

\section{References}

1. World malaria report 2020: 20 years of global progress and challenges. Geneva: World Health Organization; 2020.

2. Hemami MR, Sari AA, Raeisi A, Vatandoost $H$, Majdzadeh R. Malaria elimination in Iran, importance and challenges. Int. J. Prev. Med. 2013; 4:88.

3. Raiesi A, Nejati J, Ansari-moghaddam A, Sakeni M, Faraji L, Paktinat B, et al. Effects of foreign immigrants on malaria situation in cleared up and potential foci in one of the highest malaria burden district of southern Iran. Malar. J. 2012;11:1-1.

4. Nejati J, Tabatabaeib SM, Mozafaric E: The effect of flood and immigration on malaria situation, southeastern Iran. The first international conference of Iranian natural hazards and environmental crises, strategies and challenges (Iran ICINH) 2016.

5. Vatandoost H, Raeisi A, Saghafipour A, Nikpour F, Nejati J. Malaria situation in Iran: 2002-2017. Malar. J. 2019; 18:1-7.

6. Hanafi-Bojd A, Rafinejad J. Malaria in Bushehr Province (2001-2008), Southern Iran: An Approach Towards Elimination. J Pure Appl Microbiol. 2012; 6:565-569.

7. Hanafi-Bojd AA, Azari-Hamidian S, Hassan V, Zabihollah C. Spatio-temporal distribution of malaria vectors (Diptera: Culicidae) across different climatic zones of Iran. Asian Pac. j. trop. med. 2011; 4:498-504.

8. Mohammadkhani M, Khanjani N, Bakhtiari B, Tabatabai SM, Sheikhzadeh K. The relation between climatic factors and malaria incidence in Sistan and Baluchestan, Iran. Sage Open. 2019; 9:2158244019864205.

9. Jozaghi A, Alizadeh B, Hatami M, Flood I, Khorrami M, Khodaei N, et al. A comparative study of the AHP and TOPSIS techniques for dam site selection using GIS: A case study of Sistan and Baluchestan Province, Iran. Geosci. 2018; 8:494.

10. Nejati J, Zaim M, Vatandoost H, Moosa-Kazemi SH, Bueno-Marí R, Azari-Hamidian S, et al. Employing different traps for collection of mosquitoes and detection of dengue, Chikungunya and Zika vector, Aedes albopictus, in borderline of Iran and Pakistan. J Arthropod Borne Dis. 2020; 14:376.

11. Boshrabadi HM, Villano R, Fleming E. Technical efficiency and environmental-technological gaps in wheat production in Kerman province of Iran. Agric. Econ. 2008; 38:67-76.

12. Bakhtiari $B$, Nekooamal Kermani M, Bordbar M. Rain gauge station network design for Hormozgan province in Iran. Desert. 2013; 18:45-52.

13. Sharifi E, Steinacker R, Saghafian B. Assessment of GPM-IMERG and other precipitation products against gauge data under different topographic and climatic conditions in Iran: Preliminary results. 
Remote Sens. 2016; 8:135.

14. Basseri H, Raeisi A, Ranjbar Khakha M, Pakarai A, Abdolghafar H. Seasonal abundance and hostfeeding patterns of anopheline vectors in malaria endemic area of Iran. J. Parasitol. Res. 2010; 2010.

15. Kiani MM, Khanjankhani K, Takbiri A, Takian A. Refugees and sustainable health development in Iran. Arch. Iran. Med..2021; 24:27-34.

16. Norouzinejad F, Ghaffari F, Raeisi A. Epidemiological status of malaria in Iran, 2011-2014. Asian Pac. j. trop. med. 2016; 9:1055-1061.

17. Khan W, Rahman AU, Shafiq S, Ihsan H, Khan K. Malaria prevalence in Malakand district, the north western region of Pakistan. JPMA. 2019; 69.

18. Grietens KP, Gryseels C, Dierickx S, Bannister-Tyrrell M, Trienekens S, Uk S, et al. Characterizing types of human mobility to inform differential and targeted malaria elimination strategies in Northeast Cambodia. Sci. Rep. 2015; 5:1-12.

19. Divkolaye NSH, Burkle Jr FM. The enduring health challenges of Afghan immigrants and refugees in Iran: a systematic review. PLoS Curr. 2011; 9.

20. Wangdi K, Gatton ML, Kelly GC, Clements AC. Cross-border malaria: a major obstacle for malaria elimination. Adv. Parasitol. 2015; 89:79-107.

21. Oliveira-Ferreira J, Lacerda MV, Brasil P, Ladislau JL, Tauil PL, Daniel-Ribeiro CT. Malaria in Brazil: an overview. Malar. J. 2010; 9:1-15.

22. Inkochasan M, Gopinath D, Vicario E, Lee A, Duigan P. Access to health care for migrants in the Greater Mekong Subregion: policies and legal frameworks and their impact on malaria control in the context of malaria elimination. WHO-SEAJPH. 2019; 8:26-34.

23. Evlampidou I, Danis K, Lenglet A, Tseroni M, Theocharopoulos Y, Panagiotopoulos. Malaria knowledge, attitudes and practices among migrants from malaria-endemic countries in Evrotas, Laconia, Greece, 2013. Euro Surveill. 2015; 20:21208.

24. Nejati J, Moosa-Kazemi SH, Saghafipour A, Soofi K. Knowledge, attitude and practice (KAP) on malaria, from high malaria burden rural communities, southeastern Iran. J Parasit Dis. 2018; 42:6267.

25. Baseri H, MOUSA KS, Yosafi S, Mohebali M, Hajaran H, Jedari M. Anthropophily of malaria vectors in Kahnouj District, south of Kerman, Iran. Iran. J. Public Health. 2005; 34:27-35.

26. Nejati J, Tabatabaei SM, Salehi M, Saghafipour A, Mozafari E. Some probable factors affecting the malaria situation before and at the beginning of a pre-elimination program in southeastern Iran. $J$ Parasit Dis. 2017; 41:503-509.

27. Shahandeh K, Basseri H, Sharifzadeh Y. An application of cultural model to assess and compare malaria prevention among Afghani migrant and Baluchi resident in the endemic area, southeastern Iran. J Immigr Minor Health. 2014; 16:102-110.

28. WHO-coordinated multi-country evaluation: implications of insecticide resistance for malaria vector control. World Health Organization; 2016. 
29. Argaw MD, Woldegiorgis AG, Workineh HA, Akelom BA, Abebe ME, Abate DT, et al. Access to malaria prevention and control interventions among seasonal migrant workers: A multi-region formative assessment in Ethiopia. PloS one.2021; 16:e0246251.

30. Demissie GD, Ayele TA, Wami SD, Sisay MM, Fetene D, Wolde HF, et al. Low practice of malaria prevention among migrants and seasonal farmworkers in Metema and west Armacheho districts, Northwest Ethiopia. BMC Infect. Dis. 2021; 21:1-9.

31. Smith AD, Bradley DJ, Smith V, Blaze M, Behrens RH, Chiodini PL, et al. Imported malaria and high risk groups: observational study using UK surveillance data 1987-2006. Bmj. 2008; 337.

32. Cotter C, Sturrock HJ, Hsiang MS, Liu J, Phillips AA, Hwang J, et al. The changing epidemiology of malaria elimination: new strategies for new challenges. Lancet. 2013; 382:900-911.

33. Martins JF, Marques C, Nieto-Andrade B, Kelley J, Patel D, Nace D, et al. Malaria Risk and Prevention in Asian Migrants to Angola. Am. J. Trop. Med. 2020; 103:1918-1926.

34. Oshaghi M, Ghalandari R, Vatandoost $H$, Shayeghi M, Kamali-Nejad M, Tourabi-Khaledi H, et al. Repellent effect of extracts and essential oils of Citrus limon (Rutaceae) and Melissa officinalis (Labiatae) against main malaria vector, Anopheles stephensi (Diptera: Culicidae). Iran. J. Public Health. 2003; 32:47-52.

35. Oo WH, Cutts JC, Agius PA, Aung KZ, Aung PP, Thi A, et al. Effectiveness of repellent delivered through village health volunteers on malaria incidence in villages in South-East Myanmar: a steppedwedge cluster-randomised controlled trial protocol. BMC Infect. Dis. 2018; 18:1-10.

36. Schapira A, Zaim M, Raeisi A, Ranjbar M, Kolifarhood G, Nikpour F, et al: History of the successful struggle against malaria in the Islamic Republic of Iran. Tehran: Neekpey. 2018:27-28.

\section{Figures}

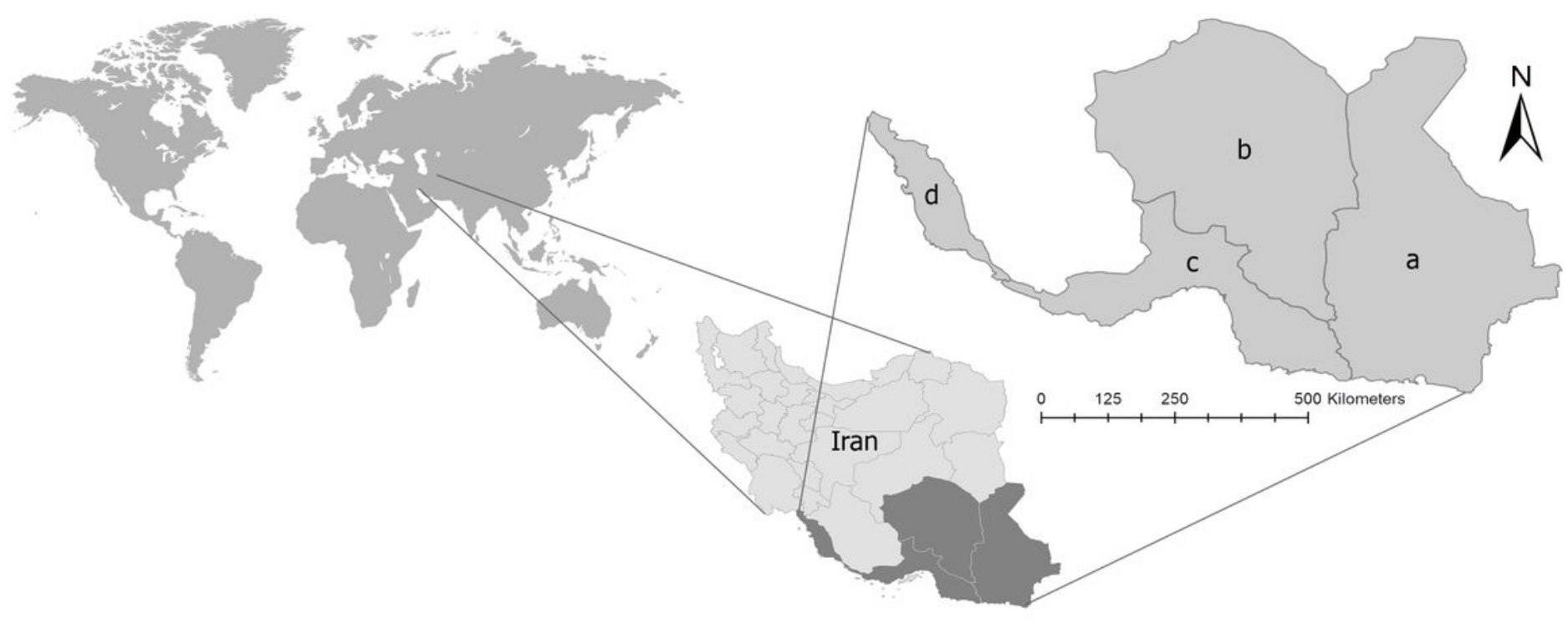

Figure 1 
Location of study area, four provinces in southern and southeastern Iran; a Sistan and Baluchestan, b Kerman, c Hormozgan, d Bushehr 